\title{
EXPLICIT DEGREE BOUNDS FOR RIGHT FACTORS OF LINEAR DIFFERENTIAL OPERATORS
}

\author{
A. BOSTAN, T. RIVOAL, AND B. SALVY
}

\begin{abstract}
If a linear differential operator with rational function coefficients is reducible, its factors may have coefficients with numerators and denominators of very high degrees. When the base field is $\mathbb{C}$, we give a completely explicit bound for the degrees of the monic right factors in terms of the degree and the order of the original operator, as well as of the largest modulus of the local exponents at all its singularities. As a consequence, if a differential operator $L$ has rational function coefficients over a number field, we get degree bounds for its monic right factors in terms of the degree, the order and the height of $L$, and of the degree of the number field.
\end{abstract}

\section{INTRODUCTION}

Context. We are interested in factorizations of linear differential operators in $\mathbb{K}(z)\left[\frac{d}{d z}\right]$, where $\mathbb{K}$ is either $\mathbb{C}$ or $\overline{\mathbb{Q}}$ (embedded into $\mathbb{C}$ ). In the latter case, there is no loss of generality in assuming that $\mathbb{K}$ is a number field (because the coefficients all live in such a number field) and in this case we denote its degree by $\kappa:=[\mathbb{K}: \mathbb{Q}]$.

Without loss of generality, we assume that $L \in \mathbb{K}[z]\left[\frac{d}{d z}\right]$, i.e., it has the form

$$
L=\sum_{j=0}^{m} p_{j}(z)\left(\frac{d}{d z}\right)^{j}
$$

for some polynomials $p_{j}(z) \in \mathbb{K}[z]$, with $p_{m} \neq 0$. The order of $L$ is $m$, and we assume that $m \geq 1$.

Assume that there exists a factorization $L=N M$ with $M, N \in \mathbb{K}(z)\left[\frac{d}{d z}\right]$, where

$$
M=\sum_{j=0}^{r} \frac{A_{j}(z)}{B(z)}\left(\frac{d}{d z}\right)^{j},
$$

for polynomials $A_{0}(z), \ldots, A_{r}(z)$ and $B(z)$ in $\mathbb{K}[z]$, with $A_{r} \neq 0$ and $B$ of minimal degree. The order of $M$ is $r$. We call degree of $M$ the quantity

$$
\operatorname{deg}_{z}(M):=\max \left(\operatorname{deg}\left(A_{0}\right), \ldots, \operatorname{deg}\left(A_{r}\right), \operatorname{deg}(B)\right) .
$$

Obviously $r \leq m$, because the order of $L$ is the sum of the orders of $N$ and $M$. But it is well known that $\operatorname{deg}_{z}(M)$ can be much larger than $q:=\operatorname{deg}_{z}(L)$, and it is in fact notoriously

Date: June 8, 2020.

2010 Mathematics Subject Classification. 11J81, 16S32, 34M15.

Key words and phrases. Differential operators, Factorization, Fuchs' relation. 
difficult to control $\operatorname{deg}_{z}(M)$ in terms of $L$. To the best of our knowledge, the first (and so far the only) bound for $\operatorname{deg}_{z}(M)$ has been given by Grigoriev [16, Theorem 1.2]. On the one hand, Grigoriev's bound holds for any factor, not only for right factors. But on the other hand, it is only an asymptotic bound; for instance, with respect to the input degree $m$, it writes $\exp \left(2^{m \cdot o\left(2^{m}\right)}\right)$ as $m \rightarrow+\infty$. One of our aims is to replace this bound by an effective bound, i.e., without any constant implicit in a $o()$ - or $O()$-estimate. This is important to ensure the termination of a recent algorithm by Adamczewski and the second author [1]; see below.

Main result. Here, we seek entirely explicit bounds holding for all $m$ and for any operator $L \in \overline{\mathbb{Q}}[z]\left[\frac{d}{d z}\right]$. As we will see, such a bound is a consequence of the following result.

Theorem 1. Let $L \in \mathbb{C}[z]\left[\frac{d}{d z}\right]$ and $M$ be a monic right factor of $L$. Then the degree of $M$ satisfies

$$
\operatorname{deg}_{z}(M) \leq r^{2}(\mathcal{S}+1) \mathcal{E}+r(\mathcal{N}+1) \mathcal{S}+r \mathcal{N}+\frac{1}{2} r^{2}(r-1)((\mathcal{S}+1)(\mathcal{N}+1)-2),
$$

where

- $r \geq 0$ is the order of $M$;

- $\mathcal{E} \geq 0$ is the largest modulus of the local generalized exponents of $L$ at $\infty$ and at its finite non-apparent singularities;

- $\mathcal{N} \geq 0$ is the largest of all the slopes of $L$ at its finite singularities and at $\infty$;

- $\mathcal{S} \geq 0$ is the number of finite non-apparent singularities of $L$.

The notions of apparent singularities, generalized exponents and slopes of a differential operator are recalled in $\S 2 . L$ is Fuchsian if and only if $\mathcal{N}=0$, in which case the generalized exponents are the usual exponents of regular or regular singular points. If $\mathcal{N} \geq 1$, then on the right-hand side of (2), the term $r \mathcal{N}$ can be replaced by $r(\mathcal{N}-1)$; see the discussion following inequality $(21)$ in $\S 2.2$. Also, the factor $(\mathcal{S}+1) \mathcal{E}$ in the first term of the righthand side of (2) can be further refined and replaced by the sum of the largest moduli of the local generalized exponents of $L$ at $\infty$ and at all its finite non-apparent singularities, rather than $(\mathcal{S}+1)$ times their maximum value. We refer the reader to the comments made after the proof of Theorem 1 concerning the choice of $\mathbb{C}$ as the base field instead of an arbitrary algebraically closed field of characteristic 0.

Bounding the degree of $M$ in terms of the degree $q$, the order $m$ and the height $H$ of $L$. Note that $r \leq m, \mathcal{N} \leq m+q$ and $\mathcal{S} \leq q$, so that Theorem 1 reduces the problem of bounding $\operatorname{deg}_{z}(M)$ to the determination of an explicit upper bound for $\mathcal{E}$, or rather for the larger quantity $E$ defined as the largest modulus of the local generalized exponents of $L$ at $\infty$ and at its finite singularities. Bounds for $E$ are known in the case where $L \in \mathbb{K}(z)\left[\frac{d}{d z}\right]$, where $\mathbb{K}$ is a number field of degree $\kappa$, embedded into $\mathbb{C}$. Grigoriev exhibited such a bound in that case, but again his result [16, Corollary, p. 21] is only asymptotic in the order $m$ of $L$, see below. The first entirely explicit bound for $E$ was obtained in 2004 by Bertrand, Chirskii and Yebbou [4]. Their approach was based in part on Malgrange's truncation method, which was eventually published in [14, pp. 97-107]. In terms of the height $H$ of 
the operator $L$ [4, p. 246 and p. 252], their bound reads

$$
E \leq 2^{(36(q+1) m \kappa)^{9(q+1)^{2} m^{3 m}}} H^{(5 \kappa(q+1) m)^{9(q+1)^{2} m^{3 m}}} .
$$

The inequalities (2) and (3), together with the bounds $r \leq m, \mathcal{N} \leq m+q$ and $\mathcal{S} \leq q$, completely solve the problem of finding an explicit upper bound for the degree of any monic right factor $M$ of $L$, when $L \in \overline{\mathbb{Q}}(z)\left[\frac{d}{d z}\right]$. It seems to be the first of this type in the literature. We have chosen to formulate Theorem 1 in terms of $\mathcal{E}$ as a parameter because the upper bound in (3) seems pessimistic and any improvement of it would implicitly improve Theorem 1. On the other hand, the other terms on the right-hand side of (2) are already polynomial in the parameters and are thus probably only marginally improvable.

Asymptotic comparisons. Below, we let $\mathcal{P}(X)$ denote different polynomials in $\mathbb{Z}[X]$, with degree and coefficients independent of $\kappa, m$ and $q$. With our notations, Grigoriev obtains the asymptotic estimate $E \leq H^{\mathcal{P}(\kappa q m)^{m}}$ as $m \rightarrow+\infty$, which is much better than (3), which reads $E \leq H^{\mathcal{P}(\kappa q m)^{q^{2} m^{3 m}}}$ as $m \rightarrow+\infty$. When $L$ is Fuchsian, Grigoriev's method as well as that of Bertrand et al. [4, p. 254] provide better bounds, which turn out to be both of the form $E \leq H^{\mathcal{P}(\kappa q m)}$; one may wonder if this is asymptotically optimal as $m \rightarrow+\infty$. In the general case, it would obviously be interesting to close the gap between the uniform bound (3) and Grigoriev's asymptotic bound for $E$. It would also be interesting to do so in intermediate cases where some properties of $L$ are known in advance. For instance, for applications related to $E$-functions (see [1]), $L$ may have only two singularities: $z=0$ which is regular, and $z=\infty$ which is irregular with slopes in $\{0,1\}$.

Optimality of the bound in Theorem 1. For any integer $k \geq 1$, the second-order operator $L:=z\left(\frac{d}{d z}\right)^{2}+(2-z) \frac{d}{d z}+k$ admits the right factor $M=\frac{d}{d z}-\frac{H^{\prime}(z)}{H(z)}$, where $H(z)$ is the confluent hypergeometric Kummer polynomial $H(z)={ }_{1} F_{1}(-k ; 2 ; z)=\sum_{\ell=0}^{k}\left(\begin{array}{l}k \\ \ell\end{array}\right) \frac{(-z)^{\ell}}{(\ell+1) !}$. Thus, $m=2, q=1, r=1$ and $\operatorname{deg}_{z}(M)=k$, and it is easy to check that $\mathcal{E}=k, \mathcal{N}=1$ and $\mathcal{S}=0$. Therefore the bound of Theorem 1 writes $\operatorname{deg}_{z}(M) \leq k$ (using the above mentioned improvement in the case $\mathcal{N} \geq 1$ ). The bound (2) is thus optimal for this example.

Degrees of left factors. Taking formal adjoints exchanges left and right factors: if $L=N M$, then $L^{\star}=M^{\star} N^{\star}$, see e.g. [20, p. 39-40]. Therefore, one can effectively bound the degree of the left factor $N$ as well, by applying Theorem 1 to $N^{\star}$ and using the fact that all the quantities (order, degree, largest slope, maximal exponent modulus, number of finite non-apparent singularities), involved in the inequality (2) for $L^{\star}$ and $N^{\star}$ can be expressed or bounded in terms of the same set of quantities for $L$ and $N$.

Minimal differential equations. Besides its own interest, one of our motivations to study this factorization problem comes from combinatorics [8] and number theory [1, 15], where certain D-finite power series in $\overline{\mathbb{Q}}[[z]]$, called $E$ - and $G$-functions, are under study. In both cases, it is useful to be able to perform the following task efficiently: given $f(z) \in \overline{\mathbb{Q}}[[z]] \backslash\{0\}$ and $L \in \overline{\mathbb{Q}}(z)\left[\frac{d}{d z}\right] \backslash\{0\}$ such that $L f(z)=0$, determine $M \in \overline{\mathbb{Q}}(z)\left[\frac{d}{d z}\right] \backslash\{0\}$ such that $M f(z)=0$ and $M$ is of minimal order with this property. Obviously, $M$ is then a right factor of $L$ and Theorem 1 applies to it. Assume $L \in \mathbb{K}(z)\left[\frac{d}{d z}\right]$ with the same data as 
above and $\mathbb{K}$ a number field, and let $f(z) \in \mathbb{K}[[z]]$ be a solution of the differential equation $L y(z)=0$. The power series $f$ need not be convergent. For any integers $r, n$ such that $1 \leq r \leq m$ and $n \geq 0$, define

$$
R(z):=\sum_{j=0}^{r} P_{j}(z) f^{(j)}(z),
$$

where $P_{j}(z) \in \mathbb{K}[z]$ are all of degree at most $n$. Then $R(z)=\sum_{k=0}^{\infty} r_{k} z^{k}$ is a formal power series in $\mathbb{K}[[z]]$, and we denote by $N$ its valuation (or order) at $z=0$, i.e., $N$ is the smallest integer $k \geq 0$ such that $r_{k} \neq 0$. A key inequality is the following upper bound on $N$ [4]: either $R(z)$ is identically zero or

$$
N \leq r(n+1)+2(q+1)^{2} m^{3}+2(q+1) m^{2}(E+1) .
$$

This is proved by putting together results by Shidlovskii [21, Lemma 8, p. 83 and Eq. (83), p. 99] and Bertrand, Chirskii, Yebbou [4, Thm. 1.2, p. 245]. With our notations, this yields $N \leq r(n+1)+n_{0}$ where $n_{0}$ is a quantity bounded above by $2(q+1) m^{2}(\mathcal{R}+1)$, with $\mathcal{R} \leq E+(q+1) m$, see [4, p. 252].

Now, given $n$ and $r+1$ polynomials $P_{j}$, not all zero, letting $N$ denote the upper bound in Eq. (4), if the first $N+1$ Taylor coefficients of $R(z)$ are all 0 , then $R(z)$ is proven identically zero, which means that $f(z)$ is a solution of

$$
M:=\sum_{j=0}^{r} P_{j}(z)\left(\frac{d}{d z}\right)^{j} \in \mathbb{K}(z)\left[\frac{d}{d z}\right] \backslash\{0\},
$$

and thus $M$ is a right factor of $L$.

This remark was used by Adamczewski and the second author [1] to give an algorithm that computes a non-zero operator $M$ such that $M f(z)=0$ and $M$ is of minimal order with this property. The input is $L \in \mathbb{K}(z)\left[\frac{d}{d z}\right]$ and sufficiently many initial Taylor coefficients of $f$, so that the following ones can be computed using $L$. Let $\widehat{n}$ be the quantity on the right-hand side of the inequality (2). The algorithm first sets $r=1$ and looks for $R$ with order $r$ and degree $\lceil\widehat{n}\rceil$ by requiring that its first $N+1$ Taylor coefficients all be 0 (this amounts to solving a homogeneous linear system with algebraic coefficients given by the Taylor coefficients of $f$ ). If no non-zero solution is found, $r$ is increased and the same procedure is repeated, and so on up to $r=m$ if necessary. In the end, $M \neq 0$ minimal for $f$ will be found.

This algorithm is not very efficient in practice. Moreover, the inequalities (3) and (4), as well as Grigoriev's Theorem 1.2 are all used to ensure the termination of the algorithm. It is important however to use Theorem 1 instead of Grigoriev's, as it holds for arbitrary differential operators $L$ and $M \in \overline{\mathbb{Q}}(z)\left[\frac{d}{d z}\right]$, and not only asymptotically. Rather than using a uniform a priori bound, a much more efficient minimization algorithm, computing tight bounds dynamically along the lines of this article and van Hoeij's work [24, Sec. 9], is under development [9]. 
Related works. The proof of Theorem 1 does not use Grigoriev's method [16], which relies on a subtle analysis of Beke's classical factorization algorithm [20, p. 118, §4.2.1], see also [22]. Instead, our method is inspired by van Hoeij's factorization algorithm [23, 24]. This algorithm internally computes, on any input operator $L$, upper bounds for the number of apparent singularities and for the degree of right factors of $L$, using the generalized Fuchs relation between local exponents. He did not give any explicit a priori degree bound, valid for any operator $L$. Our main contribution here is such a bound when the base field is a number field. It is difficult to trace back exactly when in the 80's the (generalized) Fuchs relation was found to be relevant in this type of problem. In the Fuchsian case, it was used by Chudnovsky [11] to bound the number of apparent singularities in order to obtain an effective multiplicity estimate. See also [12, p. 364, Example 2.7] for a similar use of Fuchs' relation. Chudnovsky's multiplicity estimate was adapted by Bertrand and Beukers to the general case with the help of the generalized Fuchs relation [3]. They obtained a multiplicity estimate in which the effectivity of one specific constant was not completely clear. This effectivity issue was eventually solved by Bertrand, Chirskii and Yebbou [4].

\section{Proof of TheOREM 1}

From this point on, we write $\partial_{z}$ for $\frac{d}{d z}$. Consider a monic operator

$$
R=\sum_{j=0}^{\mu} c_{j}(z) \partial_{z}^{j}=\sum_{j=0}^{\mu} \frac{U_{j}(z)}{V(z)} \partial_{z}^{j} \in \mathbb{C}(z)\left[\partial_{z}\right],
$$

with $U_{j}, V \in \mathbb{C}[z]$ and $V$ of lowest degree. We have $U_{\mu}=V, \operatorname{deg}\left(c_{j}\right):=\operatorname{deg}\left(U_{j}\right)-\operatorname{deg}(V)$ and $\operatorname{deg}_{z}(R):=\max \left(\operatorname{deg}\left(U_{0}\right), \ldots, \operatorname{deg}\left(U_{\mu-1}\right), \operatorname{deg}(V)\right)$. By definition, the set $\operatorname{Sing}(R)$ of finite singularities of $R$ is the set of roots of $V$. Amongst the finite singularities of $R$, we denote by $\alpha(R)$ the set of the apparent ones, i.e., those at which $R$ admits a local basis of power series solutions. Note that an apparent singularity is necessarily regular. We denote by $\sigma(R)$ the set of finite singularities of $R$ which are not in $\alpha(R)$, so that $\sigma(R)$ and $\alpha(R)$ form a partition of $\operatorname{Sing}(R)$. For an operator with polynomial coefficients such as $L$ from (1), the sets $\operatorname{Sing}(L), \alpha(L)$ and $\sigma(L)$ are defined as the corresponding sets for $R:=\left(1 / p_{m}\right) \cdot L$. In a factorization $L=N M$, we have $\sigma(M) \subset \sigma(L) \subset \operatorname{Sing}(L)$ but $\alpha(M)$ may have no common element with $\operatorname{Sing}(L)$. Because of this, the main difficulty in the method presented below is to bound the number of apparent singularities of a right factor of $L$.

We split the proof of the theorem into two parts. We start with the Fuchsian case because it is simpler but at the same time it contains essentially all the ideas needed to prove the general case. We refer to the book by van der Put and Singer $[20, \S 4.4]$ for the definitions of classical notions related to linear differential operators (regular singularity, Fuchsian operator, local exponents,...).

2.1. Fuchsian case. Assume that we have a factorization $L=N M$ with operators $N, M$ in $\mathbb{C}(z)\left[\partial_{z}\right]$ for which the operator $M$ is Fuchsian and monic. Note that $L$ need not necessarily be Fuchsian itself. We compute an explicit upper bound on $\operatorname{deg}_{z}(M)$ in terms 
of $\mathcal{E}$. Our strategy is inspired by van Hoeij's approach [24], itself based on ideas by Chudnovsky [11] and Bertrand-Beukers [3], see also [18].

The Fuchsianity of $M$ implies that it can be written

$$
M=\partial_{z}^{r}+\frac{A_{1}(z)}{A(z)} \partial_{z}^{r-1}+\cdots+\frac{A_{r}(z)}{A(z)^{r}},
$$

where $A(z)$ has simple roots and $\operatorname{deg}\left(A_{i}\right) \leq \operatorname{deg}\left(A^{i}\right)-i$; see [19, Chap. V, §20, p. 77]. All we now have to do is to derive an upper bound on the degree of $A$. This is done in two steps. The polynomial $A(z)$ can be factored in $\mathbb{C}[z]$ as $A(z)=A_{\text {sing }}(z) A_{\text {app }}(z)$, where the roots of $A_{\text {sing }}$ are the elements of $\sigma(M)$, while those of $A_{\text {app }}$ are the elements of $\alpha(M)$. Since $A_{\text {sing }}$ has simple roots, its degree is equal to $\# \sigma(M) \leq \# \sigma(L)=\mathcal{S}$.

The degree of $A_{\mathrm{app}}(z)$ is equal to $\# \alpha(M)$ and it can be bounded above using Fuchs' relation, which we now recall. We set

$$
S_{\rho}(M):=\sum_{j=1}^{r} e_{j}(\rho)-\frac{r(r-1)}{2}
$$

where the $e_{j}(\rho)$ 's are the local exponents of $M$ at the point $\rho$, so that clearly $S_{\rho}(M)=0$ when $\rho \in \mathbb{C} \cup\{\infty\}$ is an ordinary point of $M$. Then Fuchs' relation is the following global relation (see [20, p. 138] for a different but equivalent version):

$$
\sum_{\rho \in \mathbb{C} \cup\{\infty\}} S_{\rho}(M)=\sum_{\rho \in \operatorname{Sing}(M) \cup\{\infty\}} S_{\rho}(M)=-r(r-1) .
$$

Now, the main observation is that if $\rho \in \alpha(M)$, then $S_{\rho}(M) \in \mathbb{N} \backslash\{0\}[19$, Chap. V, $\S 18$, p. 69] ${ }^{1}$, so that

$$
\# \alpha(M) \leq \sum_{\rho \in \alpha(M)} S_{\rho}(M)
$$

and by (7) this implies that

$$
\# \alpha(M) \leq-r(r-1)-\sum_{\rho \in \sigma(M) \cup\{\infty\}} S_{\rho}(M) .
$$

Since $M$ is a right divisor of $L$, we have $\sigma(M) \subset \sigma(L)$ and for any such singularity $\rho \in$ $\sigma(M)$, the exponents of $M$ at $\rho$ are also exponents of $L$ at $\rho$, so that $\left|S_{\rho}(M)\right| \leq r \mathcal{E}+r(r-$ 1)/2 by (6). Therefore,

$$
\# \alpha(M)+r(r-1) \leq \sum_{\rho \in \sigma(L) \cup\{\infty\}}\left|S_{\rho}(M)\right| \leq(\mathcal{S}+1)\left(r \mathcal{E}+\frac{r(r-1)}{2}\right)
$$

and

$$
\# \alpha(M) \leq r(\mathcal{S}+1) \mathcal{E}+\frac{1}{2} r(r-1)(\mathcal{S}-1)
$$

\footnotetext{
${ }^{1}$ Stricto sensu, [19] proves this under an a priori stronger definition of an apparent singularity $\rho$, which requires the holomorphy of the basis of solutions at $\rho$. Note, however, that the proof is algebraic and does not use this assumption, see also [20, p. 187-188].
} 
Hence,

and finally

$$
\operatorname{deg}(A) \leq r(\mathcal{S}+1) \mathcal{E}+\frac{1}{2} r(r-1)(\mathcal{S}-1)+\mathcal{S}
$$

$$
\operatorname{deg}_{z}(M) \leq r^{2}(\mathcal{S}+1) \mathcal{E}+\mathcal{S} r+\frac{1}{2} r^{2}(r-1)(\mathcal{S}-1)
$$

This concludes the proof of Inequality (2) in Theorem 1 in the Fuchsian case, i.e., when $\mathcal{N}=0$.

2.2. General case. Again, we follow a strategy similar to that of van Hoeij [24], replacing Fuchs' relation by a generalization due to Bertrand and Laumon [6, Appendix], see also [13].

Newton polygons. Part of the information on the degrees of factors comes from patching up local information at each singularity that can be read off the Newton polygons of the operators. We first recall their main definitions and properties (see [20, p. 90, §3.3]). Let

$$
R=\sum_{j=0}^{\mu} c_{j}(z) \partial_{z}^{j}=\sum_{j=0}^{\mu} \frac{U_{j}(z)}{V(z)} \partial_{z}^{j} \in \mathbb{C}(z)\left[\partial_{z}\right],
$$

with $U_{j}, V \in \mathbb{C}[z]$ and $V$ of lowest degree and $c_{\mu}$ not necessarily equal to 1 . Consider the polynomials $P_{0}(z) \equiv 1$ and, for $j \geq 1, P_{j}(z):=\prod_{k=0}^{j-1}(z-k) \in \mathbb{C}[z]$ of degree $j$ : they are such that $\partial_{z}^{j}=z^{-j} P_{j}\left(\theta_{z}\right)$ for all $j \geq 0$, where $\theta_{z}:=z \partial_{z}$. Rewriting $R=\sum_{j=0}^{\mu} c_{j}(z) z^{-j} P_{j}\left(\theta_{z}\right)$, the Newton polygon of $R$ at 0 is obtained by taking the lowerleft boundary of the convex hull of the points $(j, i) \in \mathbb{R}^{2}$ such that the coefficient of $z^{i} \theta^{j}$ in the Laurent expansion of $R$ at $z=0$ is nonzero. The Newton polygon at another finite point $\rho \in \mathbb{C}$ is obtained similarly with $\theta_{\rho, z}=(z-\rho) \partial_{z}$ and coefficients in $\mathbb{C}((z-\rho))$, while the Newton polygon at infinity is the Newton polygon at 0 of the operator $\widetilde{R}$ obtained from $R$ by changing $z$ into $1 / z$. By definition, the slopes of the Newton polygon at $\rho$ (finite or not) are all $\geq 0$ and they are 0 if and only if $R$ is regular or regular singular at $\rho$.

In this work, we only use the largest slope of $R$ at $\rho \in \mathbb{C} \cup\{\infty\}$, that we denote by $\mathcal{N}_{\rho}(R)$; this is also known as the Katz rank of $R$ at $\rho$, see [2, pp. 229-231] and [6]. When $L=N M$, for any $\rho \in \mathbb{C} \cup\{\infty\}$, we have

$$
\mathcal{N}_{\rho}(M) \leq \mathcal{N}_{\rho}(L)
$$

Indeed, a fundamental property is that the Newton polygon of a product of operators is the Minkowski sum of their Newton polygons [20, p. 92, Lemma 3.45]. Hence, the slopes of $M$ at any point $\rho$ form a subset of those of $L$ at $\rho$.

We now assume $R \in \mathbb{C}(z)\left[\partial_{z}\right]$ to be monic and of the form (9). Let $v_{j}:=\operatorname{val}_{z=0}\left(c_{j}(z)\right)$ for $j \leq \mu$. Note that $v_{\mu}=0$. Then for any $j \in\{0, \ldots, \mu-1\}$, we have

$$
\mathcal{N}_{0}(R) \geq \frac{\left(v_{\mu}-\mu\right)-\left(v_{j}-j\right)}{\mu-j}=-1-\frac{v_{j}}{\mu-j} .
$$

It follows that for any $j \in\{0, \ldots, \mu-1\}$,

$$
\operatorname{val}_{z=0}\left(c_{j}(z)\right) \geq-\mu\left(\mathcal{N}_{0}(R)+1\right) .
$$


By a similar reasoning, for any finite $\rho \in \mathbb{C}$ and for any $j \in\{0, \ldots, \mu-1\}$,

$$
\operatorname{val}_{z=\rho}\left(c_{j}(z)\right) \geq-\mu\left(\mathcal{N}_{\rho}(R)+1\right)
$$

To deal with $\rho=\infty$, we remark that setting $\zeta=1 / z$, we have $\zeta \partial_{\zeta}=-z \partial_{z}$. Hence,

$$
\widetilde{R}=\sum_{j=0}^{\mu}\left(z^{j} c_{j}(1 / z)\right) Q_{j}\left(\theta_{z}\right)
$$

where $Q_{j}(X):=P_{j}(-X)$. In view of $\operatorname{val}_{z=0}\left(z^{j} c_{j}(1 / z)\right)=j-\operatorname{deg}\left(c_{j}(z)\right)$, the analogue of the inequality (11) is then

$$
\mathcal{N}_{\infty}(R) \geq \frac{\mu-\left(j-\operatorname{deg}\left(c_{j}\right)\right)}{\mu-j}=1+\frac{\operatorname{deg}\left(c_{j}\right)}{\mu-j}, \quad j=0, \ldots, \mu-1,
$$

leading to the bound

$$
\operatorname{deg}\left(c_{j}\right) \leq \mu\left(\mathcal{N}_{\infty}(R)-1\right), \quad j=0, \ldots, \mu-1
$$

Any finite singularity $\rho$ of $R$ is a root of $V$ and there exists $j_{\rho} \in\{0, \ldots, \mu-1\}$ such that $\rho$ is not a root of $U_{j_{\rho}}$, so that $\operatorname{val}_{z=\rho}\left(c_{j_{\rho}}(z)\right)=-\operatorname{val}_{z=\rho}(V(z))$. Using (12) with $j=j_{\rho}$, we thus deduce that

$$
\operatorname{val}_{z=\rho}(V(z)) \leq \mu\left(\mathcal{N}_{\rho}(R)+1\right)
$$

A similar reasoning at infinity gives

$$
\operatorname{deg}\left(U_{j}\right) \leq \operatorname{deg}(V)+\mu\left(\mathcal{N}_{\infty}(R)-1\right), \quad j=0, \ldots, \mu-1 .
$$

Let now $L=N M$ be a factorization of $L$ with a monic factor $M \in \mathbb{C}(z)\left[\frac{d}{d z}\right]$. We apply the bounds above to $R:=M$ and $\mu:=r$. Set

$$
\mathcal{N}=\max _{\rho \in \operatorname{Sing}(L) \cup\{\infty\}} \mathcal{N}_{\rho}(L) \quad \text { and } \quad M=\sum_{j=0}^{r} \frac{A_{j}(z)}{B(z)} \partial_{z}^{j},
$$

where the $A_{j}$ 's and $B$ are as in (9). In particular, by (10), for any $j=0, \ldots, r-1$,

$$
\begin{aligned}
\operatorname{deg}\left(A_{j}\right) & \leq \operatorname{deg}(B)+r \mathcal{N}_{\infty}(M)-r \\
& \leq \operatorname{deg}(B)+r \mathcal{N}-r .
\end{aligned}
$$

If $\rho \in \operatorname{Sing}(M)$, then Eq. (14) gives

$$
\operatorname{val}_{z=\rho}(B(z)) \leq r\left(\mathcal{N}_{\rho}(M)+1\right) .
$$

If furthermore $\rho \in \alpha(M)$, then in particular it is a regular singularity, so that $\mathcal{N}_{\rho}(M)=0$ and this bound reduces to

$$
\operatorname{val}_{z=\rho}(B(z)) \leq r
$$

Since the degree of a polynomial is the sum of the valuations (or multiplicities) at its roots, it follows from (17), (18) and $\sigma(M) \subset \sigma(L)$ that

$$
\begin{aligned}
\operatorname{deg}(B) & \leq r(\mathcal{N}+1) \cdot \# \sigma(M)+r \cdot \# \alpha(M) \\
& \leq r(\mathcal{N}+1) \mathcal{S}+r \cdot \# \alpha(M) .
\end{aligned}
$$


From (16) and (19), we see that an explicit upper bound for

$$
\begin{aligned}
\operatorname{deg}_{z}(M) & :=\max \left(\operatorname{deg}\left(A_{0}\right), \ldots, \operatorname{deg}\left(A_{r-1}\right), \operatorname{deg}(B)\right) \\
& \leq \operatorname{deg}(B)+r \mathcal{N} \\
& \leq r \mathcal{S}+r(\mathcal{S}+1) \mathcal{N}+r \cdot \# \alpha(M)
\end{aligned}
$$

will again be obtained from an explicit upper bound for $\# \alpha(M)$. (If $\mathcal{N} \geq 1$, then the right-hand side of $(20)$ can be improved to $\operatorname{deg}(B)+r(\mathcal{N}-1)$ by (16), with corresponding improvements in subsequent inequalities.)

Generalized Fuchs' relation. For any $R \in \mathbb{C}(z)\left[\partial_{z}\right]$ of order $r$, we consider the $D$-module $\widehat{R}:=\mathbb{C}(z)\left[\partial_{z}\right] /\left(\mathbb{C}(z)\left[\partial_{z}\right] R\right)$. The generalization of Fuchs' relation (7) given in $[6$, Appendice, p. 84], [7, p. 53, Theorem 2] and [13, p. 298] is

$$
\sum_{\rho \in \operatorname{Sing}(R) \cup\{\infty\}}\left(S_{\rho}(R)-\frac{1}{2} \operatorname{irr}_{\rho}(\operatorname{End}(\widehat{R}))\right)=-r(r-1),
$$

where as before

$$
S_{\rho}(R):=\sum_{j=1}^{r} e_{j}(\rho)-\frac{r(r-1)}{2}
$$

but now the $e_{j}(\rho)$ 's are the generalized local exponents of $R$ at the point $\rho \in \mathbb{C} \cup\{\infty\}$ (see [6, Appendice, pp. 82-83] or [13, p. 297] for their definition).

Given a differential operator $R$ in $\mathbb{C}(z)\left[\partial_{z}\right]$, its Malgrange's irregularity [17], denoted $\operatorname{irr}_{\rho}(\widehat{R})$, is a non-negative integer which measures the defect of Fuchsianity of $R$ at $\rho$. Next, $\operatorname{End}(\widehat{R})$ in (22) is isomorphic to the $D$-module $\widehat{R} \otimes \widehat{R^{*}}$, where $R^{*}$ is the adjoint of $R$. By [6, Appendice, p. 84], the integer $\operatorname{irr}_{\rho}(\operatorname{End}(\widehat{R}))$ can be bounded in terms of $\mathcal{N}_{\rho}(R)$ : for any $\rho \in \mathbb{C} \cup\{\infty\}$,

$$
\operatorname{irr}_{\rho}(\operatorname{End}(\widehat{R})) \leq r(r-1) \mathcal{N}_{\rho}(R) .
$$

If $\rho \in \mathbb{C} \cup\{\infty\}$ is an ordinary point or a regular singularity of $R$, we have $\mathcal{N}_{\rho}(R)=0$ and a fortiori $\operatorname{irr}_{\rho}(\operatorname{End}(\widehat{R}))=0$ as well; we thus recover the usual Fuchs relation $(7)$ when $R$ is Fuchsian.

We are now ready to bound $\# \alpha(M)$ in any factorization $L=N M$. We recall that $\alpha(M)$ and $\sigma(M)$ form a partition of $\operatorname{Sing}(M)$, and that $\sigma(M) \subset \sigma(L)$. Therefore,

$$
r(r-1)+\sum_{\rho \in \alpha(M)} S_{\rho}(M)=-\sum_{\rho \in \sigma(M) \cup\{\infty\}} S_{\rho}(M)+\frac{1}{2} \sum_{\rho \in \sigma(M) \cup\{\infty\}} \operatorname{irr}_{\rho}(\operatorname{End}(\widehat{M})) .
$$

Now, $S_{\rho}(M) \in \mathbb{N} \backslash\{0\}$ for any $\rho \in \alpha(M)$ (again by [19, Chap. V, §18, p. 69]) and $\left|S_{\rho}(M)\right| \leq r \mathcal{E}+\frac{r(r-1)}{2}$ for any $\rho \in \sigma(M) \cup\{\infty\}$ by $(23)$. 
It follows from (8) that

$$
\begin{aligned}
\# \alpha(M) & +r(r-1) \\
& \leq(\# \sigma(M)+1)\left(r \mathcal{E}+\frac{r(r-1)}{2}\right)+\frac{r(r-1)}{2} \sum_{\rho \in \sigma(M) \cup\{\infty\}} \mathcal{N}_{\rho}(M) \\
& \leq(\# \sigma(L)+1)\left(r \mathcal{E}+\frac{r(r-1)}{2}\right)+\frac{r(r-1)}{2} \sum_{\rho \in \sigma(L) \cup\{\infty\}} \mathcal{N}_{\rho}(L) \\
& \leq(\mathcal{S}+1)\left(r \mathcal{E}+\frac{r(r-1)}{2}\right)+\frac{1}{2} r(r-1)(\mathcal{S}+1) \mathcal{N} .
\end{aligned}
$$

In this sequence of inequalities, the first one follows from (24) and the second one uses (10). Hence,

$$
\# \alpha(M) \leq(\mathcal{S}+1) r \mathcal{E}+\frac{1}{2} r(r-1)(\mathcal{S}+1)(\mathcal{N}+1)-r(r-1) .
$$

It follows from (21) that

$$
\operatorname{deg}_{z}(M) \leq r^{2}(\mathcal{S}+1) \mathcal{E}+r(\mathcal{N}+1) \mathcal{S}+r \mathcal{N}+\frac{1}{2} r^{2}(r-1)((\mathcal{S}+1)(\mathcal{N}+1)-2)
$$

This completes the proof of Theorem 1.

We have mentioned above that if $\mathcal{N} \geq 1$, then the term $r \mathcal{N}$ in (26) can be replaced by $r(\mathcal{N}-1)$. It is possible to improve further this bound. Indeed, for any $R \in \mathbb{C}(z)\left[\partial_{z}\right]$, we have

$$
\sum_{\rho \in \operatorname{Sing}(R) \cup\{\infty\}}\left(\mathcal{N}_{\rho}(R)+1\right) \leq 2 \operatorname{deg}_{z}(R)+2,
$$

by the arguments used in the proof of [3, p. 185, Lemme 2bis]. Hence, the final term $\frac{1}{2} r(r-1)(\mathcal{S}+1) \mathcal{N}$ in $(25)$ could be replaced by

$$
\frac{r(r-1)}{2} \min ((\mathcal{S}+1) \mathcal{N}, 2 q+1-\# \operatorname{Sing}(L)),
$$

with a corresponding improvement of (26).

It may seem at first sight that it should be possible to adapt the proof of Theorem 1 to any algebraically closed field $\mathbb{K}$ of characteristic 0 , instead of $\mathbb{C}$. This is the case as long as equalities are used. However, the deductions made from the generalized Fuchs relation (which holds for such a field $\mathbb{K}$ ) are based on various inequalities. The argument might in principle be adapted when $\mathbb{K}$ is also endowed with an archimedean absolute value. But by Ostrowski's Theorem [10, p. 33, Theorem 1.1], such a field can be embedded into a subfield of $\mathbb{C}$ endowed with an absolute value given by a positive power of the modulus, and we would in fact gain nothing. Finally, it is not clear to us how a bound similar to (26) could be obtained with this method for a field $\mathbb{K}$ not endowed with an archimedean absolute value. We point to [24, Sec. 9] for a possible reduction of the general case to the one treated here. 


\section{ACKNOWLEDGEMENTS}

We warmly thank Daniel Bertrand for his comments on a previous version of this article, and Mark van Hoeij for a question that led us to strengthen our main result. Bruno Salvy has been supported in part by FastRelax ANR-14-CE25-0018-01.

\section{REFERENCES}

[1] B. Adamczewski, T. Rivoal, Exceptional values of E-functions at algebraic points, Bull. Lond. Math. Soc. 50.4 (2018), 697-708.

[2] D. Bertrand, Travaux récents sur les points singuliers des équations différentielles linéaires, Séminaire Bourbaki vol. 1978/79, exposés 525-542, 21 (1980), Exposé no. 538, p. 228-243.

[3] D. Bertrand, F. Beukers, Équations différentielles linéaires et majorations de multiplicités, Ann. Sci. École Norm. Sup. (4) 18.1 (1985),181-192.

[4] D. Bertrand, V. Chirskii, J. Yebbou, Effective estimates for global relations on Euler-type series, Ann. Fac. Sci. Toulouse Math. (6) 13.2 (2004), 241-260.

[5] D. Bertrand, Exposants, irrégularités et multiplicités, In Study group on ultrametric analysis, 11th year, 1983/84, pages Exp. No. 10, 6. Secrétariat Math., Paris, 1985.

[6] D. Bertrand, Exposants des systèmes différentiels, vecteurs cycliques et majorations de multiplicités, In Équations différentielles dans le champ complexe, Vol. I (Strasbourg, 1985), Publ. Inst. Rech. Math. Av., pages 61-85. Univ. Louis Pasteur, Strasbourg, 1988.

[7] D. Bertrand, On André's proof of the Siegel-Shidlovsky theorem, In Colloque Franco-Japonais: Théorie des Nombres Transcendants (Tokyo, 1998), volume 27 of Sem. Math. Sci., pages 51-63. Keio Univ., Yokohama, 1999. On-line version.

[8] A. Bostan, M. Bousquet-Mélou, M. Kauers, S. Melczer, On 3-dimensional lattice walks confined to the positive octant, Ann. Comb. 20.4 (2016), 661-704.

[9] A. Bostan, T. Rivoal, B. Salvy, An efficient algorithm for minimization of linear differential operators. In preparation.

[10] J. W. S. Cassels, Local Fields, Cambridge University Press, 360 pages, 1986.

[11] G. V. Chudnovsky, Rational and Padé approximations to solutions of linear differential equations and the monodromy theory, In Complex analysis, microlocal calculus and relativistic quantum theory (Proc. Internat. Colloq., Centre Phys., Les Houches, 1979), volume 126 of Lecture Notes in Phys., pages 136-169. Springer, Berlin-New York, 1980.

[12] O. Cormier, M. F. Singer, B. M. Trager, F. Ulmer, Linear differential operators for polynomial equations, J. Symbolic Comput. 34 (2002), 355-398.

[13] E. Corel, Relations de Fuchs pour les systemes différentiels irréguliers, C. R. Acad. Sci. Paris 333.4 (2001), Série I, 297-300.

[14] P. Deligne, B. Malgrange, J.-P. Ramis, Singularités irrégulières. Correspondance et documents, Documents Mathématiques 5, SMF, x + 188 pages, 2007.

[15] S. Fischler, T. Rivoal, Effective algebraic independence of values of $E$-functions. Preprint, 20 pages, 2019, https://arxiv.org/abs/1906.05589.

[16] D. Yu. Grigoriev, Complexity of factoring and calculating the GCD of linear ordinary differential operators, J. Symbolic Comput. 10.1 (1990), 7-37.

[17] B. Malgrange, Remarques sur les points singuliers des équations différentielles, C. R. Acad. Sci. Paris Sér. A-B 273 (1971), A1136-A1137.

[18] M. Ohtsuki, On the number of apparent singularities of a linear differential equation. Tokyo $J$. Math. 5.1 (1982), 23-29.

[19] E. G. C. Poole, Introduction to the theory of linear differential equations Dover Publications, Inc., New York, 1960. 
[20] M. van der Put, M. F. Singer, Galois Theory of Linear Differential Equations, Grundlehren der mathematischen Wissenschaften 328, xvii et 438 pages, 2003.

[21] A. B. Shidlovskii, Transcendental numbers, translated from Russian by N. Koblitz, Studies in Mathematics 12, Walter de Gruyter Co., Berlin, 1989.

[22] M. F. Singer, Liouvillian solutions of $n$th order homogeneous linear differential equations, Amer. J. Math. 103.4 (1981), 661-682.

[23] M. van Hoeij, Formal solutions and factorization of differential operators with power series coefficients, J. Symbolic Comput. 24.1 (1997), 1-30.

[24] M. van Hoeij, Factorization of differential operators with rational functions coefficients, J. Symbolic Comput. 24.5 (1997), 537-561.

Inria, Université Paris-Saclay, 1 rue Honoré d'Estienne d'Orves, 91120 Palaiseau, France

Institut Fourier, CNRS et Université Grenoble AlPes, CS 40700, 38058 Grenoble Cedex 9

Univ Lyon, Enst, UCBL, CNRS, Inria, LiP, F-69342, LyOn Cedex 07, France 